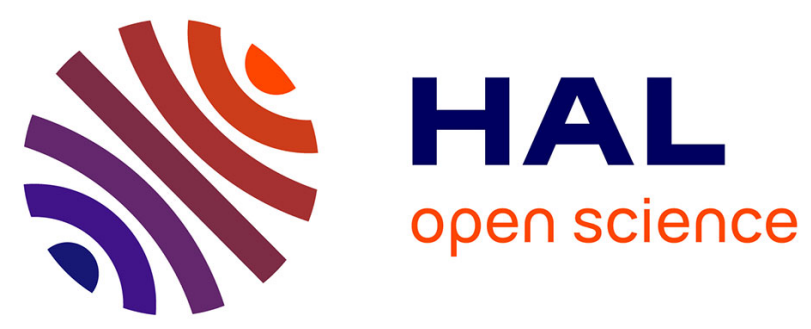

\title{
Le scénario de la répartition
}

Gerard Cornilleau, Henri Sterdyniak

\section{To cite this version:}

Gerard Cornilleau, Henri Sterdyniak. Le scénario de la répartition. Revue d'économie financière, 1992, 4 (23), pp.89-108. 10.3406/ecofi.1992.1894 . hal-00972900

\section{HAL Id: hal-00972900 \\ https: / hal-sciencespo.archives-ouvertes.fr/hal-00972900}

Submitted on 22 May 2014

HAL is a multi-disciplinary open access archive for the deposit and dissemination of scientific research documents, whether they are published or not. The documents may come from teaching and research institutions in France or abroad, or from public or private research centers.
L'archive ouverte pluridisciplinaire HAL, est destinée au dépôt et à la diffusion de documents scientifiques de niveau recherche, publiés ou non, émanant des établissements d'enseignement et de recherche français ou étrangers, des laboratoires publics ou privés.

\section{(a)(1) $\$$}

Distributed under a Creative Commons Attribution - NonCommercial - NoDerivatives| 4.0 


\title{
LE SCENARIO DE LA RÉPARTITION
}

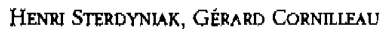

RESPECTMEMENT OFCE ET INET/ OFCE

$\mathrm{L}$

e système français de retraites publiques est l'un des plus favorables au monde. Né dans les années 30, profondément réformé à la Libération, il a été fortement amélioré depuis, notamment durant le septennat du Président Giscard d'Estaing ; aujourd'hui, les personnes âgées ne figurent plus, sauf de rares exceptions, dans la partie la plus pauvre de la population.

Le système comporte trois étages : le minimum-vieillesse relativement élevé, non-contributif, représente pour une personne seule environ $65 \%$ du SMIC net ; le régime général verse aux salariés ayant travaillé 37,5 années une pension égale à $50 \%$ de leurs dix meilleurs années de salaires bruts, plafonnés et revalorisés ; les régimes complémentaires assurent aux non-cadres à carrière complète une pension égale à $25 \%$ de leur salaire moyen brut, aux cadres $25 \%$ de leur salaire sous-plafond et $69 \%$ de leur salaire au-dessus du plafond. Un salarié qui a été payé au SMIC toute sa vie reçoit une retraite de l'ordre de $75 \%$ de celui-ci (soit $90 \%$ de son salaire net) ; un cadre qui a commencé sa carrière au plafond de la sécurité social et l' a terminée à trois fois celui-ci dispose d'une retraite de l'ordre de $55 \%$ de son demier salaire brut (soit $64 \%$ du salaire net).

En moyenne, le taux de remplacement, c'est-à-dire le rapport entre la pension et le dernier salaire net est de l'ordre de $78 \%$ pour les hommes et $72 \%$ pour les femmes (pour les salariés ayant eu une carrière complète) ${ }^{1}$. Naguère, les pensions de retraites étaient indexées automatiquement sur le salaire brut, mais depuis quelques années, la pension du régime général comme celle des régimes complémentaires n'a été indexée que sur les prix. Malgré ces défauts (multiplication des régimes complémentaires, privilèges exorbiants de certains régimes sociaux ${ }^{2}$ ), ce système est un chefd'œuvre de la social-démocratie : filet de protection pour les plus démunis, taux de remplacement important pour les bas salaires et plus faible pour les salariés ayant une certaine capacité d'épargne.

Le vieillissement de la population (baisse de la natalité, arrêt de l'imnigration, mais surtout allongement de la durée de la vie), et à un moindre

1 Selon J. Lacroix : - Les retraites en 1988, Economie et Statistique, juin 1990.

2 En particulier, l'Etat ne montre guẽre lexemple en ne payant pas de cotisaticns sur les primes des fonctionthires ef en accontant une netraike qui depend di dernier salaine, ce qui l'améne á prariquer des promotions in extremis. 


\section{REVLIE D'ECCONOMIE FINANCIËRE}

degré l'arrivée à maturité des régimes de retraites rendent nécessaire l'alourdissement des cotisations pesant sur les actifs, si l'on ne veut pas réduire les prestations.

\section{Graphique 1}

Ratio Retraités/Actifs occupés

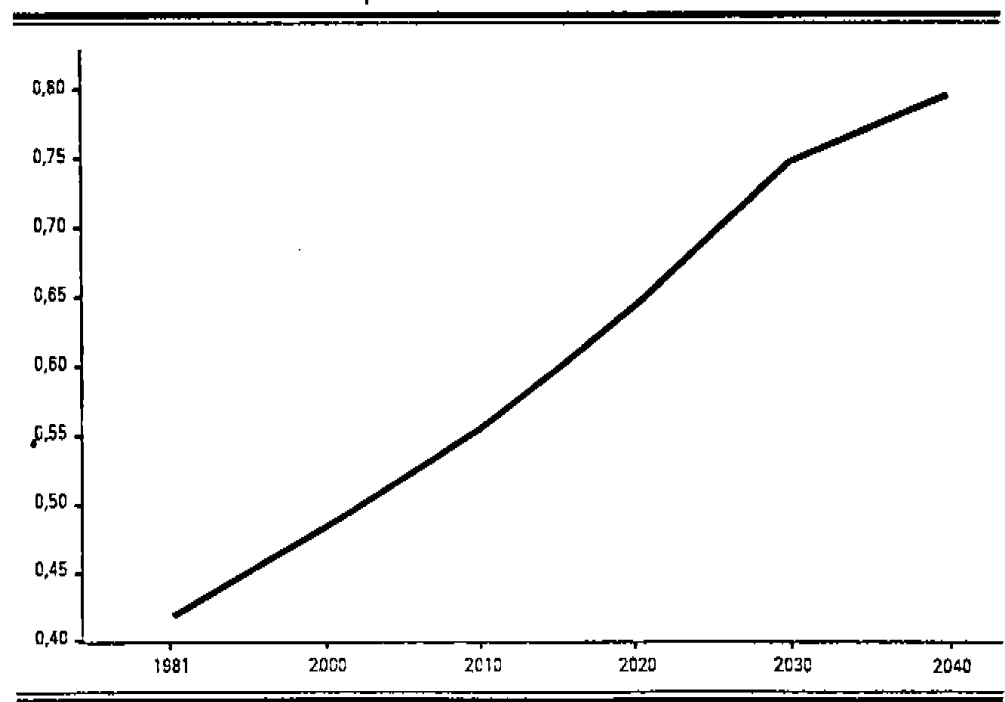

Graphique 2

Taux de cotisations sociales apparents

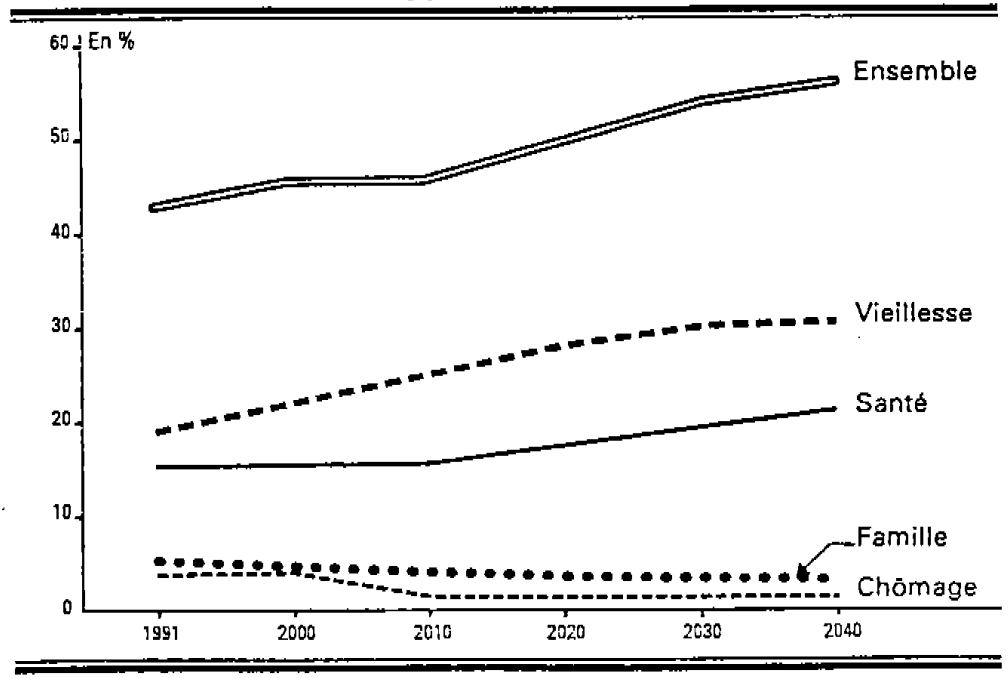




\section{La logique de la répartition...}

Le système français est, en quasi-totalité, basé sur la répartition ${ }^{3}$ : les cotisations d'une année servent à payer les prestations de la même année. Ce système repose sur la solidarité inter-générationnelle et sur un engagement moral implicite entre la Nation et les retraités : ceux-ci ont droit à une retraite satisfaisante dans la mesure où ils ont financé les retraites de la génération précédente. Au contraire, dans les systèmes de retraite par capitalisation, les caisses de retraite utilisent les cotisations pour accumuler des actifs (financiers ou immobiliers) et financent les retraites en liquidant ces actifs. La rentabilité du placement retraite est, à taux de remplacement constant, égale au taux de croissance de la productivité du travail plus celui de la population active, c'est-à-dire grosso modo aux taux de croissance du PIB. Naguère, les taux d'intérêt réels étant plus faibles que le taux de croissance du PIB, les retraites par capitalisation étaient moins avantageuses pour les individus que les retraites par répartition. Depuis 1980, c'est l'inverse, mais cette situation peut-elle se prolonger sur 20 ou 30 ans ? En régime stationnaire, la condition qui assure qu'à long terme la consommation par tête soit maximale est précisément que le taux d'intérêt réel soit égal au taux de croissance du PIB (c'est la fameuse * règle d'or *) de sorte que pour un individu, les deux systèmes sont équivalents si cette égalité est vérifiée.

Mais globalement, le système par capitalisation oblige les caisses de retraites à détenir un large portefeuille d'actifs, donc théoriquement augmente le stock de capital de l'économie, ce qui permet un niveau de production plus élevé. Ceci est la contrepartie de l'effort fait lors de la montée en charge du système de retraite : dans le système de capitalisation, la phase de démarrage est pénible, les actifs épargnent et les caisses investissent, globalement la consommation est plus faible ; dans le système par répartition, la montée en régime permet de servir des prestations retraites à des personnesn'ayant pas ou peu cotisétout en ayantinitialement des taux de cotisations très faibles : le système mange son pain blanc au départ. Dans la quasi-totalité des pays, même les plus libéraux (Etats-Unis) le système de base fonctionne selon le système de répartition parce qu'après la Seconde Guerre mondiale, les systèmes de capitalisation étaient ruinés et qu'il fallait bien foumir une retraite aux travailleurs âgés de cette époque et parce qu'il est préférable que la retraite de base ne dépende pas des fluctuations boursières et soit fixe par rapport au revenu des actifs.

Le système de retraite par répartition est nécessairement obligatoire et centralisé : siunegénération avait le choix entre répartition et capitalisation,

3 Les caisses de régimes complémentaiks ont bien des résertus, mais celles-ci ne représenent qu'un peu moins d'une ante de prestations. 
et que, compte-tenu de la rentabilité anticipée de ces deux placements, elle choisisse la capitalisation, qui paierait les retraites de la génération précédente ? En outre, il suffit qu'une partie des membres de cette génération choisisse de quitter le système par répartition pour rendre celui-ci encore moins rentable pour ceux qui restent, ce qui précipite sa disparition. La génération suivante aurait quant à elle, intérêt à revenir à la retraite par répartition puisqu'elle aurait peu de retraités de la génération précédente à entretenir. Le choix entreles deux systèmes ne peut donc être que collectif et non individuel.

De même, la solidarité inter-générationnelle doit s'effectuer dans le cadre collectif et non dans le cadre de l'entreprise, de la branche ou de la profession. Sinon, les secteurs à effectifs jeunes, en expansion rapide (la Banque hier, le personnel des collectivités locales aujourd'hui) auraient intérêt à créer des régimes autonomes, qui pourraient, compte-tenu de leur structure démographique, se contenter de faibles taux de cotisations et servir des prestations généreuses, puis, plus tard, quand ils seraient arrivés à maturité, en appelleraient à la solidarité nationale. Une telle pratique déstabiliserait le système de répartition. Pour limiter cette tendance, un système complexe de compensation démographique a été mis en place en France entre les différents régimes, mais il est source de contestations et n'est pas total. Aussi, le principe de centralisation devrait être réaffirmé ; les régimes spéciaux devraient être plus ou moins alignés sur le régime général et les régimes complémentaires unifiés.

\section{... dott fatre face au vieillissement de la population...}

Le vieillissement de la population est un phénomène général dans les pays de l'OCDE : à l'horizon 2020, les pays les plus touchés seront le Japon et le Danemark ; et, à un degré moindre l 'Allemagne, les Pays-Bas, la France ; seuls, le Royaume-Uni et l'rrlande serontrelativement épargnés(tableau 1). Dans de nombreux pays (Etats-Unis, Japon, Allemagne), des réformes du système public de pensions ont déjà étê entreprises ; elles combinent généralement une réduction du niveau des pensions, un report de l'âge du départ à la retraite, soit immédiat soit programmé pour les années à venir, une hausse des cotisations de façon à permettre aux caisses d'engranger des excédents. Ces mesures posent problème : la réduction des pensions est difficile à envisager pour les bas salaires ; le report de l'âge de la retraite ne peut avoir lieu que dans une situation de plein-emploi, sinon on perd en prestations chômage ce que l'on gagne en prestations retraites; les excédents des caisses n'ont de sens que si l'épargne nationale supplémentaire correspondante est effectivement investie dans le secteur productif et non si, comme aux Etats-Unis, ils permettent à l'Etat de financer sa dette et 
d'atteindre plus facilement l'objectif de déficit public, car dans ce cas, les générations futures devront rembourser la dette publique au lieu de payer des cotisations sociales supplémentaires, ce qui revient au même. On peut pourtant tirer des leçons des exemples étrangers : il faut planifer sur 4050 ans l'évolution des recettes et des dépenses de l'assurance-vieillesse pour prendre en douceur les toumants nécesaires ; les individus doivent être informés à l'avance du niveau de retraites que le régime public leur garantit ; chaque régime doit fixer ses dépenses et ses recettes de façon à être actuariellement équilibré sur cette période de temps, compte-tenu de perspectives raisonnables et révisables d'évolution de la population totale, de la population active et des rémunérations.

Tableau1.

Rappont entre le nombre de personnes agées et la population active potentielle (en \%)

Population de plus de 65 ans/population de 15 à 65 ans.

\begin{tabular}{|l|c|c|c|c|c|c|}
\hline & 1980 & 1990 & 2000 & 2010 & 2020 & 2040 \\
Belgique & 21,9 & 21,2 & 21,9 & 23,6 & 26,8 & 36,0 \\
Eanemark & 22,2 & 22,5 & 21,5 & 24,3 & 36,5 & 42,0 \\
France & 17,2 & 19,4 & 21,9 & 22,9 & 25,3 & 38,2 \\
Grèce & 21,9 & 20,9 & 23,4 & 24,5 & 30,7 & 38,2 \\
Irlande & 20,5 & 18,3 & 22,7 & 25,8 & 27,4 & 34,0 \\
Italie & 18,2 & 18,5 & 16,9 & 16,4 & 18,7 & 27,1 \\
Pays-bas & 20,7 & 20,2 & 22,6 & 25,7 & 29,4 & 41,1 \\
Portugal & 17,4 & 18,4 & 18,8 & 22,0 & 28,9 & 42,0 \\
RFA & 16,2 & 17,9 & 20,7 & 21,3 & 23,6 & 33,3 \\
Royaume-Uni & 23,4 & 22,3 & 25,4 & 30,5 & 33,5 & 48,5 \\
Japon & 23,3 & 23,0 & 22,3 & 22,7 & 25,5 & 33,1 \\
Etats-Unis & 13,5 & 16,2 & 22,6 & 29,5 & 33,6 & 37,8 \\
\hline
\end{tabular}

Source : OCDE, Le vieillissement démographique, 1988. 


\section{... mais l'augmentation des taux de cottsattons peut être rendue} acceptable par la crotssance

L'augmentation des taux de cotisations retraites sera-t-elle supportable? Pour aborder cette question, le raisonnement démographique est insuffisant : une population riche et peu touchée par le chômage aura moins de mal à financer les retraites de ses parents, même s'ils sont plus nombreux à rester en vie longtemps, qu'une population qui s'appauvrit.

Pour juger du caractère supportable de l'augmentation des charges liées aux retraites, nous avons projeté à l'horizon 2040, par pas de 10 ans, les revenus et l'ensemble des prestations sociales, en supposant qu'aucune grande réforme ne survienne (voir annexe). Deux modifications du système actuel ont toutefois été prises en compte. La première concerne le financement de l'augmentation de la charge des caisses de retraite supposé peser à l'avenir, sur les seuls actifs, sans que les entreprises soient mises à contribution, afin de ne pas nuire à l'emploi et à la compétitivité. L'arbitrage que font collectivement les travailleurs entre revenu d'activité, âge du départ à la retraite et niveau des pensions ne doit pas influencer le coût salarial global : les travailleurs ne doivent pas avoir l'illusion que les retraites sont financées par les entreprises sans coût pour eux. Les hausses nécessaires de cotisations doivent être prélevées sur les seuls revenus des personnes couvertes.

La seconde modification concerne le niveau des retraites : l'objectif doit être de garantir aux retraités un niveau de revenu fixe par rapport à celui des actifs : le montant des retraites doit donc être explicitement indexé sur le montant des salaires nets, tant en ce qui concerne le mode de calcul de la pension des nouveaux retraités que la revalorisation des retraites déjà liquidées. Ce n'est pas le cas actuellement : le mode de liquidation des pensions fait que celles-cisuivent théoriquement le salaire brut ; l'indexation des pensions est maintenant discrêtionnaire. Cette réforme nécessite une modification du code de la Sécurité sociale, de sorte que la pension corresponde non pas à $50 \%$ du salaire brut mais à $60 \%$ du salaire net, et que les pensions déjà liquidées soient indexées sur le salaire net ${ }^{4}$. Ceci permettrait de clarifier les droits des retraités qui seraient assurés que le niveau relatif de leur pension ne peut être mis en cause par des chocs conjoncturels ou structurels.

4 Lindexation des retraites liquides demait être rélisée à l'aide d'un indice de salaire net à structure constonse. L'abjectif est en effet d'assuner la parité entre l'évolution de la retraite et celle du salaine de réfenence utilisé au moment de la liquidation. Il ny a donc pas lieu de tenir compte de la dérive du salaire mojen lièe à la deformation des qualifications (GVT).

Uhe reforme équivalente à lindexation sur les salaines nets, contristerait à mainsenit l'indexation des retraites sur les salaines bruts, mais ầ augmenter les cotisations portant sur les retraitế chaque fois que f'on augmente les cotisations portont sur les actiff. Mais cela seroit instifunionnellement delicat dans le cas de conisations cbômage ef vieillexpe. 
Sous ces hypothèses le maintien du système de répartition nécessite que, chaque année, de 1991 à 2040 , le taux de cotisations sociales 5 augmente d'environ 0,3 point (tableau 3). Ceci reste relativement acceptable si le salaire brut croît chaque année de $2 \%$ comme la productivité moyenne du travail ; mais la croissance du salaire net ne serait que de $1,2 \%$ entre 2010 et 2030 et n'atteindrait que $1,6 \%$ après 2030. Par comparaison, de 1970 à 1991, le taux apparent de cotisations sociales a augmenté de 0,6 point par an, ce qui fait que les salaires nets par tête n'ont augmenté en moyenne que de 1,5\% par an contre 2,4 pour les salaires y compris cotisations. En 2040, le taux de prélèvement sur les revenus d'activité serait de $55,7 \%$ contre 42,6 actuellement : chaque actif devrait dépenser 3,7 fois plus qu'aujourd'hui pour la protection sociale mais son revenu brut sera 2,8 fois son revenu actuel ; une fois déduites ses cotisations sociales, son revenu net sera encore 2,1 fois son revenu net actuel. Si l'effort nécessaire est important, il n'est donc nullement insupportable, ce que masque le seul raisonnement démographique qui fait abstraction de la croissance économique. Certains ont prétendu que le système de retraite par répartition ferait peser un poids écrasant sur les générations futures, du fait de l'alourdissement nécessaire des cotisations, qu'il était donc inéquitable ; mais il est malaisé de faire des arbitrages intertemporels consistant à comparer le poids supporté par des générations successives. L'accumulation du capital et surtout celle des connaissances et du progrèstechnique ont fait, du moins depuis deux siècles en Europe, que les fils ont toujours été plus riches que leurs pères. Le ralentissement démographique fera aussi que la charge d'enfants supportée par les actifs de demain sera plus faible. Au total, le caractère inique ou insupportable de cette situation reste à démontrer.

Pourtant même si la croissance globale des taux de cotisations peut sembler supportable à très long terme, le passage des années 2000 à 2030 est délicat. Au cours de cette période, il serait très difficile de faire face aux accidents conjoncturels. Un certain lissage de la hausse des taux de cotisations est donc souhaitable.

\section{Assurer la pérennité du système}

La société française est donc placée devant un arbitrage délicat : maintenir le système tel qu'il est grasso modo (compte tenu des réformes que nous avons suggérées) ou le transformer en profondeur en introduisant une dose plus ou moins forte de capitalisation. Nous allons explorer successivement les deux hypothèses.

5 II s'agit du toux apporent ou sens du tableau 3. 
Le maintien du système à l'identique aboutit en 2030 à un taux de cotisations de $55,7 \%$ contre 42,6 actuellement. D'aucuns affirment que ce taux deviendra insupportable et que les actifs chercheront à y échapper, soit socialement, soit individuellement. Par exemple, si les travailleurs expriment leurs revendications salariales en termes de salaire net, ces cotisations augmenteront le coût du travail et les entreprises rechigneront à s'installer en France ; de même, les cadres pourront choisir de travailler dans un pays où le système social est moins développé. En fait, ces risques doivent être relativisés : la Sécurité sociale ne représente pas seulement une charge pour le travailleur, mais aussi un avantage important : grâce à ce système, les travailleurs sont assurés contre le chômage, ont une assurance-maladie et une retraite, de sorte que l'entreprise doit compenser ces avantages pour tout travailleur expatrié. Le vieillissement de la population, le poids grandissant des dépenses médicales sont des phénomènes généraux dans la quasi-totalité des pays de l'OCDE. Le coût de la couverture contre ces différents risques sera grosso modo le même quel que soit le système public ou privé. Par exemple, en ce qui conceme les retraites, pour disposer à 60 ans d'une pension de l'ordre de $75 \%$ du salaire net, c'est compte tenu de l'espérance de vie actuelle des travailleurs, une cotisation de l'ordre de $22,3 \%$ du salaire d'activité qu'il faudra payer quel que soit le système et ce chiffre ne peut qu'augmenter avec l'allongement des durées de vie.

Les ménages tiennent à la possibilité d'acquérir un actif relativement sûr comme un droit proportionnel au pouvoir d'achat des salaires, plutôt qu'à faire reposer leurs retraites uniquement sur des actifs financiers qui, obligataires, leur feraient courir le risque de l'inflation ou, sous forme d'actions, le risque d'un krach boursier, cela d'autant plus qu'ils peuvent acquérir des actifs financiers par ailleurs grâce à leur épargne personnelle. De 1968 à 1978, par exemple, le pouvoir d'achat des salaires nets a augmenté de 3,4\%l'an tandis que les ménages qui auraient acquis des titres obligataires en 1968 , auraient subi une perte de pouvoir d'achat de $1,2 \%$ l'an. Imagine-t-on ce qu'auraient été les fluctuations de la consommation si une part importante des retraites avait dépendu des cours de la Bourse ?Or il n'y a que l'Etat qui puisse garantir un actif assis sur le pouvoir d'achat du salaire.

Mais le niveau élevé des taux de cotisations sera d'autant mieux ressenti que la compétitivité, donc la rentabilité, des retraites publiques, sera grande et assurée clairement... Ceci passe par des changements de forme (bien faire apparaître sur les bulletins de paye l'ensemble des cotisations retraites comme un salaire différé et non un prélèvement obligatoire, renseigner clairement les salariés chaque année sur les droits acquis) et par des réformes de fond : les droits acquis par les cotisants doivent être 
garantis contractuellement, le contrat devant comporter une règle de liquidation déterminée et l'indexation des pensions sur le salaire net, de sorte que les droits à la retraite soient aussi sûrs juridiquement que les actifs financiers.

Rien ne serait plus dangereux dans cette optique que le projet parfois évoqué, de désengagement de l'Etat, celui-ci prenant en compte les prestations non-contributives er laissant les partenaires sociaux gérer à leur discrétion le reste du système : l'évolution des prestations dépendrait de façon imprévisible pour les retraités des rapports de force et des alliances entre syndicats ouvriers et patronaux ; en période de difficultés économiques, les retraites seraient réduites, les règles de liquidation seraient remises en cause ${ }^{6}$; la fiabilité de l'actif * droit à la retraite " serait faible ; les cotisations seraient mal ressenties puisque de contreparties aléatoires.

\section{Rédutre le coût du système}

On peut par ailleurs penser à deux pistes pour réduire le coût du système. L'allongement de l'âge de départ à la retraite est la plus simple. En régime stationnaire, un système de retraite à 60 ans (et $75 \%$ de taux de remplacement net) nécessite une ponction sur les salaires d'activité de l'ordre de $22,3 \%$; une retraite à 65 ans de seulement $16,6 \%$, ce qui règle donc une grande partie des problèmes. Certes, un tel report ne pourrait être envisagé que dans une situation de plein-emploi - il ne serait, en effet, ni économiquement justifié, ni socialement acceptable de diminuer le nombre de retraités pour augmenter celui des chômeurs - donc selon notre projection pas avant 15 ans ; d'ailleurs, dans ce cas, le report se ferait plus ou moins naturellement : les entreprises, qui auraient du mal à embaucher, chercheraient à retenir leurs salariés âgés.

Ce serait d'autant plus acceptable que la part des emplois physiquement pénibles est en forte diminution et donc que les travailleurs atteignent moins * usés * l'âge de 60 ans. En sens inverse, la retraite à 60 ans permet souvent aux entreprises d'être débarassées sans drames de certains trávailleurs vieillissants, moins capables de s'adapter au progrès de la technique et surpayés en raison des règles d'ancienneté : le maintien de travailleurs âgés est, dans certains cas, un coût pour l'entreprise. Par ailleurs, la retraite à 60 ans peut apparaître comme une utilisation normale des gains de productivité, comme un choix social entre loisir et consommation. Pour permettre à chacun de choisir plus librement, l'idéal serait une retraite à la carte, c'est-à-dire actuariellement neutre entre 60 et 65 ans, mais cette réforme aurait un impact différent selon l'âge à partir duquel serait assuré un niveau satisfaisant de retraite : si le taux de remplacement de $70 \%$ est

6 Suivant le (mautris) exemple des prestations gérîs par l'UNEDIC. 
atteint à 65 ans, les gens partant à la retraite à 60 ans n'auraient un taux de remplacement que de $52 \%$ ce qui est faible et relativement dissuasif ; si le taux de remplacement de $70 \%$ est atteint à 60 ans, les gens partant à la retraite à 65 ans, devraient toucher, dans l'optique dela neutralité actuarielle, $92 \%$ de leur salaire net, ce qui serait très coûteux. Une solution intermédiaire semble préférable : si le taux de remplacement de $70 \%$ est assuré à 62,5 ans, un salarié partant à la retraite à 60 ans touchera $60 \%$ de son salaire net ; $81 \%$ s'il part à 65 ans. Par contre, il serait peu avisé de trop augmenter la durée de cotisations ouvrant le droit à la retraite à taux plein. Si on veut qu'un cadre, qui a commencé à travailler à 24 ans (âge normal pour le niveau $\mathrm{Bac}+5$ ) puisse partir à la retraite à 62 ans, cette durée ne peut être supérieure à 38 ans ; une durée de 42 ans, comme le propose le CNPF, le dissuaderait de partir avant 66 ans. On peut douter de l'intérêt pour les entreprises d'un nombre important de cadres vieillissants.

Supposons, à titre illustratif, qu'à partir de 2010, l'âge légal de la retraite passe de 60 à 62 ans. Cette mesure serait alors doublement positive puisqu'elle permettrait à la fois de stimuler la croissance en desserrant la contrainte de plein emploi (la production serait plus élevée de 3,2\% en 2040) et de réduire la charge du régime d'assurance-vieillesse. La hausse nécessaire du taux global de cotisations serait réduite de 1,7 point. Certe mesure, associée à une reprise de l'immigration, permettrait donc une réduction sensible de l'augmentation des charges de la protection sociale.

Une autre façon de réduire la montée en charge des prestations retraites, serait de diminuer quelque peu le montant des taux de remplacement. Limitons-nous à une baisse qui ne remettrait pas en cause le principe du système. Cette baisse devrait, malheureusement mais obligatoirement, porter sur l'ensemble des retraités, et non sur les plus riches, qui ont déjà des taux de remplacement relativement bas. Le plus simple serait de profiter de la réforme nécessaire du système d'indexation pour décider que la pension du régime général serait désormais de $55 \%$ du salaire net (au lieu de $60 \%$ comme le voudrait le maintien du niveau actuel). Ceci permettrait une baisse de 2,1 points du taux de cotisations en 2040. Une modification, décidée une fois pour toutes, serait préférable à un grignotage du montant relatif des pensions pour la sous-indexation des revalorisations annuelles. Cette dernière a deux inconvénients: elle rend incertaine l'évolution des retraites futures; elle diminue petit à petit le niveau de vie relatif des retraités les plus âgés, ce qui n'est guère loyal. Par contre, certaines des réformes étudées par le CGP ${ }^{7}$ seraient peu avisées : faut-il remettre en cause les avantages non contributifs accordées aux personnes ayant élevé au moins 3 enfants alors qu'il reste nécessaire de favoriser la

7 Voir . Vérillir solikLaine , Commissariat Gënüral au Plan, Paris, 1986. 
natalité ? Il serait peu judicieux de passer à un système par points pour le régime général ; un système mixte où les prestations dépendent à la fois des meilleurs années (régime gênéral) et de l'ensemble de la carrière (régime complémentaire) a l'avantage de ne pas trop pénaliser les personnes à carrière ascendante ; le renforcement de l'indexation sur le salaire net ${ }^{8}$ dans le cadre du régime actuel garantirait ipso facto que les retraités prennent leur juste part de la croissance ou des difficultés économiques sans qu'il soit besoin de rajouter un degré de liberté pour l'Etat (la fixation de l'évolution du point), donc une incertitude supplémentaire pour les retraités.

\section{Uttliser la capttalisatton?}

Explorons maintenant l'autre solution : le développement des retraites par capitalisation. Elle consisterait à bloquer les taux de cotisations retraite au niveau actuel de $20 \%$ du revenu d'activité et à ajuster à l'avenir le système en diminuant le taux de remplacement. A l'horizon 2040, c'est une baisse de l'ordre d'un tiers du niveau relatif des retraites qui serait nécessaire : le taux de remplacement assuré par les retraites de répartition passerait de 75 à $50 \%$. Mais une telle baisse ne peut être décidée sans préavis, ni précaution. Une telle réforme devant être annoncée à l'avance pour permettre aux actifs de se constituer un supplément de retraite, elle doit l'être maintenant pour ne s'appliquer pleinement que dans 30 ou 40 ans. Il faut, en même temps, mettre sur pied un système de retraite par capitalisation, avec un avantage fiscal équivalent à celui de la retraite par répartition (exonération des fonds investis et taxation de la pension servie) s'il s'agit d'un placement tunnel, c'est-à-dire où les fonds investis sont totalement immobilisés et ne peuvent être libérés que sous la forme d'une rente viagère.

Pour que ce système se développe, le gouvernement doit indiquer clairement que le taux de remplacement des retraites par capitalisation diminuera fortement à l'avenir. Sinon, les actifs d'aujourd'hui considéreront que le contrat moral actuel tient toujours et ne seront donc pas inciter à se constituer une retraite supplémentaire. Ce régime nouveau devrait être financé par les seules personnes concernées pour ne pas augmenter les charges des entreprises. La réforme aurait l'inconvénient de créer un troisième régime de retraite complêmentaire. Ce régime devrait être obligatoire; sinon, il risque de n'être adopté que par les cadres; on se retrouverait dans 30 ans avec de profondes inégalités entre les retraités et un abaissement inacceptable du niveau de vie relatif des retraités non-

\footnotetext{
8 Ure partie de la bausse du salaire moken s'explique en fait par la bausse du niveau mojen de qualification de la main-d'cetute. A ussi peut-on penser que les retraites detraient être inderées sur le salaire net moyen à qualification constande qui progresse en mojen the de 0.5 point de moins par an (le GVT soldè) que le salaire moyen.
} 
cadres. Etudions donc les modalités possibles, les coûts et les avantages de cette réforme.

Pour maintenir au niveau actuel le taux de cotisations des retraites par répartition, il faudrait créer un régime de capitalisation auquel dès à présent les travailleurs devraient souscrire au taux de $10 \%$ de leur revenu tout en continuant de souscrire au régime de répartition à un taux de $20 \%{ }^{9}$. Ce régime accumulerait au départ de forts excédents et atteindrait un régime d'équilibre vers 2030. La réforme ne touchera pas les travailleurs nés avant 1930, qui sont déjà en retraite actuellement. Si le taux d'intérêt réel est peu différent du taux de croissance de l'économie et comme un régime de répartition est identique, en régime permanent, à un régime de capitalisation, la création de ce régime n'aurait pas d'impact pour les travailleurs nés aprés 2010 : ils paieront 20 points de cotisations au régime par répartition et 10 au régime par capitalisation au lieu de payer 30 points au régime par répartition sans la réforme ; leur retraite leur sera payée pour $50 \%$ de leur salaire net par le régime de répartition; pour $25 \%$ par le régime de capitalisation.

Par contre, les générations nées entre 1930 et 2010 seraient perdantes dans l'opération puisqu'elles devraient payer immédiatement une cotisation au taux de $30 \%$ alors que, en laissant les choses de l'état, la haussẻ des cotisations de 20 à $30 \%$ ne serait que progressive (comme indiqué dans le tableau 3). Ces générations intermédiaires auraient des retraites assurées par le cumul des deux régimes, la part des retraites par capitalisation croissant au cours du temps pour atteindre un tiers en 2030. Ces générations seraient donc condamnées à un surcroît d'épargne par rapport à la situation sous réforme, surcroût nécessaire pour assurerl'accumulation des réserves des caisses de capitalisation. Le montant du capital nécessaire des caisses de capitalisation en 2030 serait de l'ordre de 4 fois le revenu net des actifs, soit de 1,2 fois le PIB, soit encore $55 \%$ du capital net actuel de l'économie française.

Du point de vue individuel, la mise en place du système de capitalisation ne diminue donc pas le taux de prélèvement nécessaire pour obtenir un taux de remplacement donné ; au contraire, elle l'augmente pendant les quarante années de transition. La différence toujours au niveau individuel est qu'une partie des retraites futures n'est plus gagée par le contrat implicite que représente le système de retraites par répartition, mais par un titre, ce qui fournit une certaine garantie aux futurs retraités contre les aléas politiques mais augmente leur vulnérabilité aux chocs boursiers ou inflationnistes. Mais, la réforme se heurte à court terme à plusieurs problèmes,

9 On peut imaginer une montée en régime moins brutale, mais dans ce cas la part des organismes de capitalisation senait plus faible à terme et le taux des cotisations par répartition se stabiliserait à un niveau plus étere. 
en plus de ses conséquences macroéconomiques que nous discuterons plus loin : elle sera extrêmement impopulaire : comment faire admettre aux salariés de perdre d'un coup $10 \%$ de leur revenu sur la base de prévisions macroéconomiques contestables ? Quel gouvemement s'y risquerait ${ }^{10}$ ? Par ailleurs, les salariés réagiraient en diminuant leur épargne propre, de sorte que l'épargne supplémentaire expost serait plus faible que l'épargne ex ante. De plus, les ménages répugnent à s'engager dans un placement de retraite, c'est-à-dire de longue durée et en sortie obligatoire en rente viagère; ils préfêrent conserver une certaine liberté pour la jouissance de leur patrimoine, pouvoir investir dans l'immobilier et laisser un héritage. Une formule de retraites par capitalisation facultative risque d'avoir peu de succès une formule obligatoire, de faire beaucoup de mécontents.

Comment seraient gérés ces fonds ? Plusieurs formules sont concevables. Certains préconisent que ces fonds soient gérés au sein des entreprises : ils leur apporteraient ainsi des quasi-fonds propres et leur permettraient d'augmenter leurs investissements. Ces fonds pourraient être de type obligatoire ou être des titres de participation au capital. Dans le premier cas, ils seraient rémunérés par l'entreprise au taux du marché ; ce n'est un avantage pour elle que si elle ne trouve pas des fonds auprès du système bancaire ou sur les marchés, donc si elle est peu solide et dans ce cas, ce sont les salariés qui supportent le risque. Dans le second cas, de même, le salarié est amené à supporter involontairement des risques quel'entreprise ne réussit pas à faire assumer par le marché. Il place dans le même panier son travail et son capital, ce qui n'est guère optimal.

Une formule de retraite centrée sur l'entreprise peut sembler favorable à court terme, mais elle est dangereuse à long terme : les entreprises peuvent faire faillite ; les fonds peuvent être détournés ; le ratio retraités/ salariés est bas en période de forte croissance de l'entreprise (où le système est favorable pour elle et où celle-ci peut prendre des engagements inconsidérés) et s'alourdit en période de stagnation où l'entreprise doit faire appel à l'Etat pour tenir ses engagements (voir le cas de la SNCF et des mines hier ; des banques demain) ; certains retraités seront gagnants, ayant eu la chance d'appartenir à des secteurs porteurs ; d'autres perdants, s'ils travaillaient dans des secteurs en déclin. Les fonds de pensions ne sont assimilables à des fonds propres (et ne peuvent donc contribuer à favoriser l'investissement) que si les retraités passent après les autres créanciers de

10 On remarquera por exemple que dans son projet - Pour une bause du salaire dired, Droit social, fermier 1992, E. Balladur propose de compenser Le surcrofl d'epargne demande aux travailleurs par uthe bohusse des salaines, elle-mế ne financēep par une réduction des cotisations employeurs, elle-mémefinancée en dernier ressont par une bypothē ique - tationalisation des dépenses publiques , sans plus de précision. Cette nationalisation $n$ est en tout état de c. wse pas de l'ordre de grandew des fonds mécessaines. 
l'entreprise en cas de difficultés économiques et si leurs pensions dépendent des résultats des entreprises. Si les retraités sont des créanciers privilégiés et si leurs retraites sont indexées sur les salaires, cette dette est de caractère obligatoire et n'est pas spécialement rentable pour les entreprises. Pas de miracle... les fonds de pensions ne peuvent être une dette spécialement avantageuse pour les entreprises que s'ils constituent un actif peu rémunérateur ou risqué pour les salariés. Reste la deuxième solution : les fonds seraient collectés et placés par des OPCVM ou des sociétés d'assurances.

Le problème essentiel est alors l'impact macroéconomique de cette épargne supplémentaire. Pourcertains économistes, la France (et lemonde) manque d'épargne et singulièrement d'épargne longue. L'instauration des retraites par capitalisation comblerait ce manque, les entreprises pourraient investir plus facilement, le capital donc la production serait plus fort demain et les retraites, même celles par répartition, pourraient ainsi être financées plus facilement. Le débat sur les retraites nous ramène donc au débat sur la pénurie d'épargne, l'idée sous-jacente à des projets étant que le manque d'épargne bloque actuellement l'investissement, de sorte qu'il suffirait d'encourager l'épargne des ménages pour obtenir ipso facto une hausse de l'investissement. Mais, on ne voit guère par quels canaux cette augmentation aurait lieu. Que l'investissement dépende essentiellement de la demande anticipée ou des profits, une hausse de l'épargne des ménages, c'est-à-dire une baisse de la consommation, entraînerait une diminution de la demande qui provoquerait une baisse plutôt qu'un essor de l'investissement, que ce soit par diminution des débouchés ou du fait de la baisse des profits en période de baisse de l'activité. Le seul canal favorable serait celui d'une baisse des taux d'intérêt. Mais une hausse du taux d'épargne n'aurait guère en soi d'impact sur les taux d'intérêt français largement déterminés par l'extérieur.

- Par ailleurs, n'est-il pas paradoxal de demander aux ménages d'augmenter leur épargne, donc leurs actifs, alors que l'Etat souhaite réduire la dette publique et que les entreprises souhaitent se désendetter, compte tenu du niveau élevé des taux d'intérêt réels ? On peut certes espérer accumuler des avoirs sur l'étranger : les besoins d'investissement ne manquent pas à l'échelle mondiale, mais on voit mal les caisses de retraites prendre le risque de prêter aux PVD ou même aux pays de l'Est, du moins sans garantie publique. Quelle serait la situation des retraités si leur retraite dépendait des remboursements de l'Irak, de la Pologne ou du Mexique ? Le souvenir de l'emprunt russe est toujours vivace. On peut penser à diversifier les placementsà l'étranger, dans les zones sûres du monde développé (comme le font les Japonais ou les Allemands), mais comment avoir un excédent commercial important pour financer ces sorties de capitaux ? Celui-ci ne 
peut résulter, à compétitivité donnée, que d'une baisse de la demande, donc de l'activité en France. Cette baisse favorisera certes la * désinflation compétitive - mais au prix, à court terme, d'une nouvelle hausse du chômage.

Faut-il utiliser le système des retraites pour résoudre le problème de l'équilibre macroéconomique alors que le gouvernement dispose d'autres armes de politique économique, plus facilement actionnables car ne mettant pas en jeu des engagements sur 40 ou 60 ans ? Raisonnons donc dans le cadre d'une stratégie macroéconomique optimale. Le gouvernement choisitun taux d'intérêt objectif : celui-ci pouvant être soit déterminé, comme aujourd'hui parl'extérieur, soit parun certain arbitrage intertemporel s'il s'agit d'un pays dominant. Il choisit le niveau souhaitable de production, celui-ci pouvant correspondre soit au taux de chômage naturel, soit à un certain chômage nécessaire pour faire pression sur les salaires. On en déduit un niveau de demande nécessaire, celui-ci requiert un certain déficit global (ou excédent) des administrations. Imaginons que nous restons dans la situation actuelle : le taux d'intérêt est certes trop élevé mais le gouvernement français n'a aucun moyen de le faire baisser, ce niveau ne résultant pas d'une pénurie d'épargne en France, mais de facteurs internationaux. Augmenter les prélèvements sur les ménages pour constituer un - trésor - pour plus tard se traduirait plus par une chute du niveau de l'activité et de l'investissement que par l'essor de l'investissement en capital physique, qui serait le seul moyen de transférer dans le temps du pouvoir d'achat. Si on compense cette chute de l'activité par un déficit budgétaire accru, les générations futures auront à payer le remboursement de la dette publique et sa charge, ce qui revient au même que de payer des retraites supplémentaires. Il est inutile et même nuisible, de chercher à favoriser l'épargne lorsque la croissance est faible et qu'on ne dispose pas des marges de manœuvre permettant de la stimuler.

Une forte impulsion donnée à l'épargne des ménages ne pourrait avoir des effets bénéfiques que comme élément d'accompagnement, dans une deuxième phase, d'un plan de relance dela croissance par l'investissement, par exemple, à la suite d'une forte baisse des taux d'intérêt en Europe. Ce n'est qu'au moment où une croissance trop vive vient buter sur les capacités de production, en machines ou en hommes, qu'une hausse de l'épargne est nécessaire et utile pour la poursuite de la croissance. Mais, notons deux points : dans ce cas, la rentabilité des placements de capitalisation retomberait au niveau de la rentabilité des placements de répartition. Une forte baisse des taux d'intérêt réels est nécessaire pour que les entreprises acceptent de s'endetter plus, mais elle rend moins rentable la retraite par capitalisation. D'autre part, là aussi, le développement des caisses de capitalisation se ferait à la place d'une hausse des impôts portant sur les 
ménages, donc au prix d'une hausse du déficit public. La seule différence, c'est qu'on peut penser qu'il serait mieux accepté psychologiquement mais cela est douteux si les cotisations aux caisses de capitalisation sont obligatoires.

Reste le dénouement de l'opération : une partie importante des retraites dépendrait de la Bourse ou des taux d'intérêt, dont l'évolution n'est pas certaine : quand les régimes de retraites voudront réaliser leurs actifs pour servir les droits acquis, ceci risque davantage d'entraîner une chute de la Bourse ou une forte inflation que la réduction de l'investissement qui serait nécessaire pour réorienter la production vers les biens de consommation.

\section{Constituer des résenves?}

Mais une troisième voie, qui a notre préférence, est possible. Ce serait que, en restant dans le cadre de la retraite par répartition, les caisses constituent des réserves afin de lisser l'évolution des taux de cotisations et de réduire la variabilité du taux de rendement des cotisations. Cela implique que les régimes de retraite accumulent des excédents d'aujourd'hui à 2010 pour limiter la hausse nécessaire dans les années 2010 à 2030. Mais, il sera difficile, en une période où la Sécurité sociale a déjà des problèmes de financement, de faire admettre que les caisses de retraites soient excédentaires pour préparer un avenir hypothétique. Là aussi, se pose le problème du bouclage macroéconomique : il faut éviter que ces excédents ne pèsent sur l'activité. L'excédent nécessaire des caisses de retraites par répartition doit donc s'ajouter au déficit macroéconomiquement nécessaire ainsi que nous l'avons défini plus haut : par exemple, s'il est nécessaire que l'ensemble des administrations ait un déficit de $1 \%$ du PIB et que les caisses de retraites souhaitent avoir un excédent de $2 \%$, les autres administrations devront avoir un déficit de $3 \%$.

Naturellement, ceci n'est utile ques'il est macroéconomiquement nécessaire que l'ensemble des administrations ait un déficit, parce qu'il est plus facile de justifierun excédent des caisses de retraite que de l'Etat, donc dans le cas où ex ante la demande est excédentaire, au taux d'intêrêt que le gouvernement choisit. Le plus simple serait que cet excédent soit affecté au financement de certaines dépenses d'investissement de l'Etat : grands travaux exceptionnels (comme la contruction d'universités, d'autoroutes, de TGV), dotation des entreprises publiques, financement de certains investissements dans les pays du Sud ou de l'Est.

La dette de l'Etat envers les caisses constituerait alors une dette publique supplémentaire qui aurait l'avantage d'être indexée sur les salaires et non sur le taux d'intérêt ; ce serait avantageux pour l'Etat dont certaines recettes sont elles-mêmes en fait indexées sur les salaires. Cette formule permettrait 
une programmation à long terme de la hausse des cotisations sociales; dans les années 2010-2030, les marges dégagées permettraient d'éviter que l'on soit contraint d'augmenter trop fortement les taux de cotisations précisément dans une période de faible croissance économique potentielle. Cette solution serait globalement proche de la mise en place d'un système de retraite par capitalisation obligatoire, mais les pensions continueraient à être garanties par l'Etat.

Assurer la pérennité de notre système de retraite par répartition est souhaitable. Elle est possible en accumulant un grand nombre de réformes ponctuelles : indexation des retraites sur le salaire net, prise en charge des hausses de cotisations par les seuls actifs et non par les entreprises, gestion rigoureuse des régimes spéciaux tendant à leur alignement sur le régime gênéral ; puis, après 2010, si, comme on peut l'espérer, le plein-emploi est de retour, réouverture contrôlée des frontières à l'immigration, léger report de l'âge de départ à la retraite et instauration d'une plus grande liberté de choix de l'âge de départ : enfin d'ici 2010 , augmentation lente et régulière des taux de cotisations salariés pour permettre aux caisses de retraites par répartition d'accumuler un certain excédent et pour éviter une montée trop brutale des taux de cotisations.

Pour choisir entre les systèmes possibles, il ne faut ni s'adonner au fétichisme du taux de prélèvement obligatoire le plus bas possible, ni utiliser les retraites pour réglerun autre problème : celui du financement de l'économie. La privatisation de la protection sociale, et celle du financement des retraites, pourraient avoir des conséquences sociales plus graves que l'augmentation des taux de cotisations. Elles réintroduiraient des inégalités sociales face à la maladie et à la viẹillesse, que le système actuel, fondé sur la solidarité, permet de contrôler.

\section{ANNEXE \\ L'EQUILIBRE DES CAISSE DE RETRAITE ET DE SÉCURITÉ SOCIALE À L'HORIZON 2040}

Pour mesurer l'ordre de grandeur de l'effort nécessaire au financement des retraites à long terme il faut approcher les projections démographiques et économiques pour déterminer la part de leurs revenus que les ménages devront consacrer aux dépenses de retraites.

Les variables démographiques ont été déterminées à partir d'une projection par 
âge de la population totale, basée sur le recensement de 1990, en supposant la stabilisation du taux de fécondité à 1,9 (soit 0,1 de plus que le taux actuellement observé pour tenir compte de l'effet probable de la tendance au décalage des naissances). Les tAUx de mortalité sont supposés évoluter dans le prolongement des tendances observées depuis 1975, ce qui conduit à un allongement important des durées de vie. Le solde migratoire est supposé constant sur l'ensemble de la période et égal à 50000 par an, soit sa valeur actuelle. Compte tenu de la dérive des taux d'activité (hausse des taux féminins, prolongement de l'allongement de la scolarité quiretarde l'âge d'entrée dans la vie active, stabilisation, puis légère reprise des taux d'activité après 55 ans due à l'amélioration de la situation du marché du travail), la population active potentielle continuerait à croitre jusqu'en 2010 avant de diminuer légèrement par la suite.

En ce qui concerne les variables économiques, nous avons retenu, jusqu'en 2010, une hypothèse tendancielle de croissance de la production de $3 \%$ par an. Les gains annuels de productivité du travail serait de $2,5 \%$ jusqu'en 2000 avant de décroître de 2000à 2010. En 2010 l'économie française atteindrait le plein emploi (conespondant en fait à un taux de chômage de $5 \%$ ) ; la croissance serait ensuite limitée par les gains de productivité et l'évolution de la population active potentielle. Compte tenu de la baisse de la population active potentielle, la croissance de l'économie française après l'an 2010 serait très faible de l'ordre de 1,8\% l'an (tableau 3). L'emploi total, évoluant donc comme les ressources en main-d'cuvre, baisserait de $0,2 \%$ par an en moyenne de 2010 à 2040 . Le nombre de retraités, ramenté à la population active occupée, augmenterait très fortement passant de 0,42 retraités par actif occupé en 1990 à 0,48 en 2000 et 0,79 en 2040 (grapbique 1).

Le niveau des retraites serait à l'avenir, indexé sur les salaires nets de façon à assurer un taux de remplacement constant. Mais l'arrivée à maturité dés régimés de retraites, le fait que les travailleurs (en particulier les femmes) sont de plus en plus nombreux à avoir effectué une carrière complète entraîne une croissance un peu plus rapide des retraites moyennes que celle du salaire moyen. Le chiffrage de cette dérive est délicat, car, en contrepartie, le nombre de bénéficiaires du minimum vieillesse, de pensions de réversion, de majoration pour conjoints à charge diminue. Enfin, la raréfaction des familles nombreuses diminue le nombre de bénéficiaires de bonifications à ce titre. D'ailleurs, l'observation des années récentes montre que le pouvoir d'achat de la retraite moyenne n'augmente pas plus vite que celui du salaire net moyen : la dérive n'apparaît plus depuis 1983. Ceci est dû au fait que les pensions ont été nettement sous-indexées par rapport au salaire moyen brut, et même par rapport au salaire moyen net : la perte est de l'ordre de $2,5 \%$ pour les pensions du régime général (tableau 2); de plus, la dérive entre l'évolution du plafond de la Sécurité sociale et le coefficient de revalorisation des salaires fait qu'il devient impossible à un salarié, même ayant toujours cotisé au plafond de toucher la pension maximum du régime général " ${ }^{11}$. Nous avons supposé qu'à l'avenir la sous-indexation des retraites prendrait fin et avons retenu l'hypothèse d'une dérive positive d'un demi-point par an pendant encore 20 ans. Au total le pouvoir d'achat de la retraite moyenne croît donc comme celui du salaire moyen net augmenté de $0,5 \%$ par an jusqu'en 2010

En ce qui concerne les autres prestations sociales, nous avons indexé le pouvoir d'achat par tête des prestations familiales et des prestations chômage sur le salaire net. Nous avons tenu compte d'une croissance autonome des dépenses réelles de santé par tête de 2,5\% par an et du fait que les dépenses de santé des personnes

11 Ceci est signale dans le rapport de fêrier 1991 de la Commission des comples de la Sécuritè sociale. 
Tableau 2. Evolution de 1985 à 1990 des prix, des salaires et des pensions de retraites.

\begin{tabular}{|l|c|}
\hline Prix à la consommation & $16,5 \%$ \\
SMIC brut : & $21,1 \%$ \\
net : & $16,2 \%$ \\
Salaire moyen brut : & $23,8 \%$ \\
net : & $18,8 \%$ \\
Plafond de la Sécurité Sociale & $22,8 \%$ \\
Pensions du régime général * & $16,3 \%$ \\
Minimum vieillesse & $16,3 \%$ \\
Point de l'UNIRS ** & $18,9 \%$ \\
Point de l'AGIRC & $15,9 \%$ \\
\hline
\end{tabular}

* Le coefficient de revalorisation des salaires pour le calcul de la pension évolue comme les pensions.

** Représentatif des caisses complémentaires des non-cadres.

âgées de plus de soixante ans sont en moyenne le double de celles des moins de soixante ans.

Jusqu'en 2000 l'augmentation du taux de cotisation vieillesse, dueà la croissance du nombre des retraités mais aussią la poursuite de la dérive des retraites moyennes par rapport aux revenus d'activité, explique l'essentiel de la montée du taux de cotisation global (tableau 3 et graphique 2). De 2000 à 2010, les taux de l'assurance vieillesse continuent à croitre mais cette fois essentiellement du fait de l'arrivée à l'âge de la retraite des classes nombreuses de l'après-guerre. De même le taux de cotisation à l'assurance maladie voit sa progression accélérée par le vieillissement de la population. En sens inverse le taux de cotisation chômage diminue du fait du retour au plein emploi. Après 2030 , le taux vieillesse est à peu près stabilisé et seul le taux santé continue à augmenter.

Notre projection suppose le ralentissement du rythme de croissance tendanciel de la productivité du travail qui passerait de 2,5 à $2 \%$ après l'an 2000 . Toutefois, la raréfaction de la main-d'œuvre et le retour au plein emploi peuvent, associés au progrès technique, entraîner un mouvement de rationalisation de la production plus rapide, qui pourrait permettre au moins de maintenir la croissance de la productivité autour de $2,5 \% \mathrm{par}$ an. Dans ces conditions, le financement des prestations sociales serait facilité à partir de 2010, par une croissance économique plus forte. Les taux de cotisations correspondant aux prestations indexées sur les revenus d'activité (retraites, famille, chốmage) ne seraient pas affectés, mais lés taux santé augmenteraient moins. Au total le taux de cotisations global pourrait être 
REVUE D'ÉCONOMIE FINANCIËRE

Tableau 3. Revenus d'activités et prestations sociales à l'horizon 2040

\begin{tabular}{|c|c|c|c|c|c|c|}
\hline & 1991 & $\begin{array}{l}2000 / \\
1991\end{array}$ & $\begin{array}{l}2010 / \\
2000\end{array}$ & $\begin{array}{l}2020 / \\
2010\end{array}$ & $\begin{array}{l}2030 / \\
2020\end{array}$ & $\begin{array}{l}2040 / \\
2030\end{array}$ \\
\hline Taux de croissance du PIB & - & 3,0 & 3,0 & 1,9 & 1,8 & 1,8 \\
\hline $\begin{array}{l}\text { Taux de croissance du } \\
\text { salaire moyen net de } \\
\text { cotisations sociales }\end{array}$ & - & 1,9 & 2,0 & 1,2 & 1,2 & 1,6 \\
\hline $\begin{array}{l}\text { Taux de cotisations } \\
\text { apparents (en fin de } \\
\text { période) }{ }^{*}:\end{array}$ & & & & & & \\
\hline Vieillesse & 18,8 & 21,5 & 24,7 & 27,7 & 29,8 & 30,3 \\
\hline Santé & 15,1 & 15,3 & 15,3 & 17,1 & 19,2 & 20,8 \\
\hline Famille & 5,0 & 4,6 & 4,1 & 3,7 & 3,5 & 3,3 \\
\hline Chômage & 3,8 & 4,0 & 1,5 & 1,4 & 1,3 & 1,2 \\
\hline Total & 42,6 & 45,5 & 45,5 & 49,8 & 53,7 & 55,7 \\
\hline
\end{tabular}

- Les taux de cotisations calculés ici sont des taux apparents qui comparent le montant des prestations sociales au total des revenus d'activité des ménages (qui représentent en $199160 \%$ du PIB). Ils ne sont donc pas directement comparables aux taux institutionnels qui s'appliquent aux salaires bruts de cotisations salariés et nets de cotisations employeurs.

abaissé de 2 points en 2040 par rapport au niveau projetế précédemment $(53,7$ au lieu de $55,7 \%$ ). Mais surtout, les revenus d'activité pourraient augmenter plus rapidement et la croissance des salaires nets par tête serait mieux assurée à long terme : de 1990 à 2040 elle serait de $2 \%$ en moyenne et, au point le plus bas, entre 2020 et 2030, elle ne descendrait pas en-dessous de $1,8 \%$ (rappelons que de 1970 à 1991, elle ne fut que de 1,6 ), alors qu'elle n'atteindrait que $1,2 \%$ avec une productivité croissant de $2 \%$. L'effort demandé aux actifs serait donc nettement atténué et son acceptation plus vraisemblable.

Une fois notre pays retourné au plein-emploi, l'attitude à l'égard de l'immigration peut changer. La France qui était traditionnellement largement ouverte aux flux migratoires, reste un des pays d'Europe les moins peuplés. Supposons qu'à partir de 2010, le solde migratoire annuel pase à 75000 ce qui correspond à la moyenne des années 1954/1975, contre 50000 actuellement. Ceci permettrait à l'horizon 2040, un PIB plus élevê de 3,6\% et un niveau plus bas de 0,8 point du taux de cotisations apparent, qui n'augmenterait que de 12,2 points entre 1991 et 2040 au lieu de 13 points. Le recoursà une immigration plus forte, dans un contexte de plein emploi, serait donc une solution particulièrement intéressante : elle permet d'accélérer la croissance économique tout en allégeant les charges de la protection sociale pendant une période très longue. En effet, les immigrés sont en moyenne plus actifs et plus jeunes que l'ensemble de la population. 